\title{
Tratamento das manifestações orais da doença enxerto contra hospedeiro crônica: revisão sistemática da literatura
}

\author{
Treatment of oral manifestations of chronic graft versus host disease: systematic literature review
}

\author{
Felipe Souza Lima Alencar \\ Álvaro Cavalheiro Soares \\ Programa de Residência Multiprofissional em Oncologia, Instituto Nacional de Câncer José Alencar Gomes da Silva (INCA), Rio de Janeiro, Brasil \\ Héliton Spindola Antunes \\ Divisão de Ensaios Clínicos e Desenvolvimento de Fármacos, Instituto Nacional de Câncer José Alencar Gomes da Silva (INCA), Rio de Janeiro, Brasil \\ - Os autores declaram que não há conflito de interesse.
}

Resumo

Objetivo: Sintetizar as principais modalidades terapêuticas utilizadas no tratamento das manifestações orais da doença enxerto contra hospedeiro crônica (DECHc), através de uma revisão sistemática da literatura. Material e Métodos: Foi realizada uma busca nas bases de dados Cochrane Library, Dentistry \& Oral Sciences e Pubmed, com descritores para o tratamento da DECHc com envolvimento oral, entre os meses de Junho e Outubro de 2015. Definiu-se uma metodologia de seleção a partir da leitura dos títulos, resumos e artigos disponíveis. Resultados: Foram encontradas 32 publicações, que apresentaram um total de 13 fármacos imunossupressores, sendo 07 dos quais foram empregados por meio de terapia local e 06 via sistêmica. Dois artigos tratavam de terapias auxiliares para 0 alívio da hipossalivação. Conclusão: As medicações encontradas podem servir de parâmetros para futuros fármacos a serem empregados na prática clínica. 0 tratamento da DECHc oral ainda apresenta eficácia limitada, todavia, sugere-se o uso de medicação tópica para reduzir efeitos sistêmicos. São necessários estudos prospectivos, randomizados e controlados para comprovar a eficácia de certas medicações.

Palavras-chave: terapia; doença enxerto-hospedeiro; imunossupressores; esteroides; manifestações bucais.

\section{Abstract}

Objective: To summarize the main therapeutic modalities used in the treatment of oral manifestations of graft versus host disease (GVHD), through a systematic review of the literature. Material and Methods: A search was conducted in the databases Cochrane Library, Dentistry \& Oral Sciences and Pubmed, with descriptors for the treatment of chronic GVHD with oral involvement, between June and October 2015. The methodology was defined from reading the titles, abstracts and articles available. Results: Founded 32 publications that presented a total of 13 immunosuppressant drugs, and 07 which were employed by local therapy and systemically 06 . Two articles treated auxiliary therapies to relieve hyposalivation. Conclusion: Drugs found may serve as parameters for future drugs to be used in clinical practice. The treatment of oral GVHD still has limited effectiveness, however, suggests the use of topical medications to reduce systemic effects. Prospective studies are needed, randomized controlled trials to prove the effectiveness of these medications.

Keywords: therapy; graft-versus-host disease; immunosuppressants; steroids; oral manifestations.

\section{Introdução}

A doença enxerto contra hospedeiro crônica (DECHc) é a principal causa de morbimortalidade pós-transplante de medula óssea. ${ }^{1}$ A etiologia é decorrente da reação das células do doador (enxerto) contra o organismo do paciente (hospedeiro). ${ }^{2}$ Seus sintomas podem ocorrer em um único órgão ou podem estar disseminados, sendo comuns alterações em pele, olhos, boca, trato gastrointestinal, pulmões e articulações. ${ }^{1-3}$

A mucosa oral é a segunda localização mais comum da DECHc. ${ }^{4}$ Clinicamente, as lesões são semelhantes ao Líquen Plano (reticulado ou erosivo), apresentando áreas eritematosas com estrias hiperceratóticas, comumente encontradas em mucosa jugal e língua. ${ }^{5}$ Outros sinais encontrados, embora não sejam diagnósticos da DECHc oral são: áreas atróficas, úlceras recobertas por pseudomembrana e microstomia, associada à esclerose dérmica de áreas periorais. Reações semelhantes às encontradas em lesões autoimunes podem acometer glândulas salivares, resultando em redução da função e consequentemente hipossalivação e xerostomia. Complicações indiretas da DECHc oral são relacionadas com terapia local a longo prazo com corticoides, as principais são doença periodontal e infecções oportunistas, como candidíase, cárie e doença periodontal. ${ }^{6}$

O manejo da doença oral é essencial para aliviar os sinais e sintomas desta doença, manter as funções orais e restaurar a integridade da mucosa minimizando as complicações. ${ }^{7} \mathrm{O}$ tratamento tópico com esteroides tem sido a medicação de escolha para o manejo da DECHc oral, oferecendo vantagens como menos efeitos sistêmicos ou potencialização do tratamento sistêmico adjuvante. Embora haja vantagens, não existem evidências sobre qual agente é o mais eficaz. ${ }^{8,9}$ Dessa forma, a escolha do corticoide a ser prescrito é norteada pelo conjunto de sinais e sintomas que o paciente apresenta. ${ }^{8}$ Devido aos efeitos adversos causados pela terapia com corticoides a longo prazo, há estudos com terapias secundárias não farmacológicas como o uso da radiação ultravioleta $\mathrm{B}^{10,11}$ e laserterapia. ${ }^{12}$

Pacientes com DECH coral têm mais risco de desenvolver cáries devido à hipossalivação, que gera a falta de proteção pela saliva, sendo necessário o uso de estimulantes salivares, como gomas de mascar sem açúcar e agentes enzimáticos em forma de colutórios. A manutenção de uma boa saúde oral e o efetivo controle de placa bacteriana é importante para reduzir os riscos de infecção local. O uso de fluoretos em gel podem ser indicados em casos de xerostomia severa para prevenir 
cáries rampantes. ${ }^{9-13}$ Protocolos clínicos para manejo das escleroses periorais ainda são escassos e a experiência clínica é baseada em estudos não limitados a mucosa oral. ${ }^{4}$

Este estudo tem como objetivo sintetizar as principais modalidades terapêuticas da DECHc oral, além de discutir sua eficácia, através de uma revisão sistemática da literatura, entre os meses de junho e outubro de 2015. Dessa maneira, esse trabalho pretende contribuir para um maior conhecimento do manejo clínico das lesões, além de fornecer aos cirurgiões-dentistas e outros profissionais de saúde um panorama sobre o tema. No tocante à assistência, acredita-se que essa publicação possa auxiliar para uma melhor qualidade de atendimento e de vida aos pacientes com DECHc oral.

\section{Material e Métodos}

Realizou-seuma revisão sistemática da literatura buscando esclarecer as principais terapêuticas utilizadas para o tratamento da DECHc oral. Em um primeiro momento, foi elaborada a pergunta de pesquisa de acordo com a estratégia PICO, definindo o problema a ser pesquisado, as intervenções descritas na literatura, estabelecendo medidas comparativas e o desfecho esperado. Nesse momento, foram definidos os descritores usados, identificação dos termos relacionados aos componentes da estratégia de busca. Em seguida, foi realizada uma busca bibliográfica nas bases de dados: Dentistry \& Oral Sciences, Pubmed, Cochrane Library (Cochrane Reviewse Cochrane Trials).

A pesquisa foi direcionada para a localização de descritores nos títulos, resumos e MeSH das publicações com auxílio de operadores booleanos (Tabela 1). A busca ocorreu entre os meses de junho e outubro de 2015. A publicação mais antiga datou de 1997 e a mais recente em 2015. A retirada das referências duplicadas e a exportação dos registros foram realizadas no gerenciador de referências Endnote webซ.

A metodologia aplicada para seleção das publicações baseou-se na ferramenta para realização de revisão sistemática de literatura PRISMA (Figura 1). Na seleção inicial foram identificadas as publicações através da leitura dos títulos e resumos. Os critérios de inclusão foram publicações que abordassem o tratamento das manifestações orais da DECHc nos idiomas inglês, espanhol e português. Os critérios de exclusão foram trabalhos realizados em animais ou abordagem terapêutica sem envolvimento oral. Na etapa de triagem, foram incluídos nos fatores de exclusão estudos que não obtiveram acesso à leitura na íntegra.

Foram extraídos das publicações, dados referentes ao tipo de desenho de estudo. Para os estudos envolvendo fármacos, separou-se por tipo de droga usada, via de administração, mecanismo de ação, resposta em cavidade oral e efeitos colaterais encontrados. Das pesquisas com administração sistêmica, foi extraído o percentual das respostas global e oral. Esses dados foram expressos em tabelas elaboradas com o auxílio do software Microsoft Word 2007 (Microsoft Corporation, Redmond, WA).

Tabela 1. Estratégias de busca nas bases de dados Pubmed, Dentistry \& Oral Sciences e Cochrane Library

\begin{tabular}{|c|c|c|c|}
\hline Base de dados & \multicolumn{2}{|r|}{ Estratégia de busca } & Resultados \\
\hline PubMed & \multicolumn{2}{|r|}{$\begin{array}{l}\text { ((GVHD[tiab] OR Graft vs Host Disease[tiab] OR Graft vs } \\
\text { Host Disease[mh]) AND (therapy[tiab] OR treatment*[tiab] } \\
\text { OR treating[tiab]) AND (Oral Manifestations[tiab] OR Oral } \\
\text { Manifestations[mh] OR Dentist*[tiab] OR Dentists[mh] OR } \\
\text { Mouth[tiab] OR bucal[tiab])) }\end{array}$} & $\mathrm{n}=70$ \\
\hline $\begin{array}{l}\text { Dentistry \& Oral } \\
\text { Sciences }\end{array}$ & \multicolumn{2}{|r|}{$\begin{array}{l}\text { (GVHD OR Graft vs Host Disease OR Graft vs Host Disease) AND } \\
\text { (therapy OR treatment OR treating) AND (Oral Manifestations OR } \\
\text { Oral Manifestations OR Dentistry OR Dentists OR Mouth OR bucal) }\end{array}$} & $n=25$ \\
\hline \multirow{10}{*}{ Cochrane Library } & & Search Hits & \multirow{10}{*}{$\begin{array}{l}\text { COCHRANE } \\
\text { TRIALS } \\
\mathrm{n}=39\end{array}$} \\
\hline & \#1 & GVHD or Graft vs Host Disease & \\
\hline & \#2 & MeSH descriptor: [Graft vs Host Disease] this term only & \\
\hline & \#3 & \#1 or \#2 & \\
\hline & \#4 & Oral Manifestations or Dentist* or Mouth or bucal & \\
\hline & \#5 & MeSH descriptor: [Oral Manifestations] this term only & \\
\hline & \#6 & MeSH descriptor: [Dentists] this term only & \\
\hline & \#7 & $\# 4$ or \#5 or \#6 & \\
\hline & \#8 & therapy or treatment* or treating & \\
\hline & \#9 & \#3 and \#7 and \#8 & \\
\hline
\end{tabular}




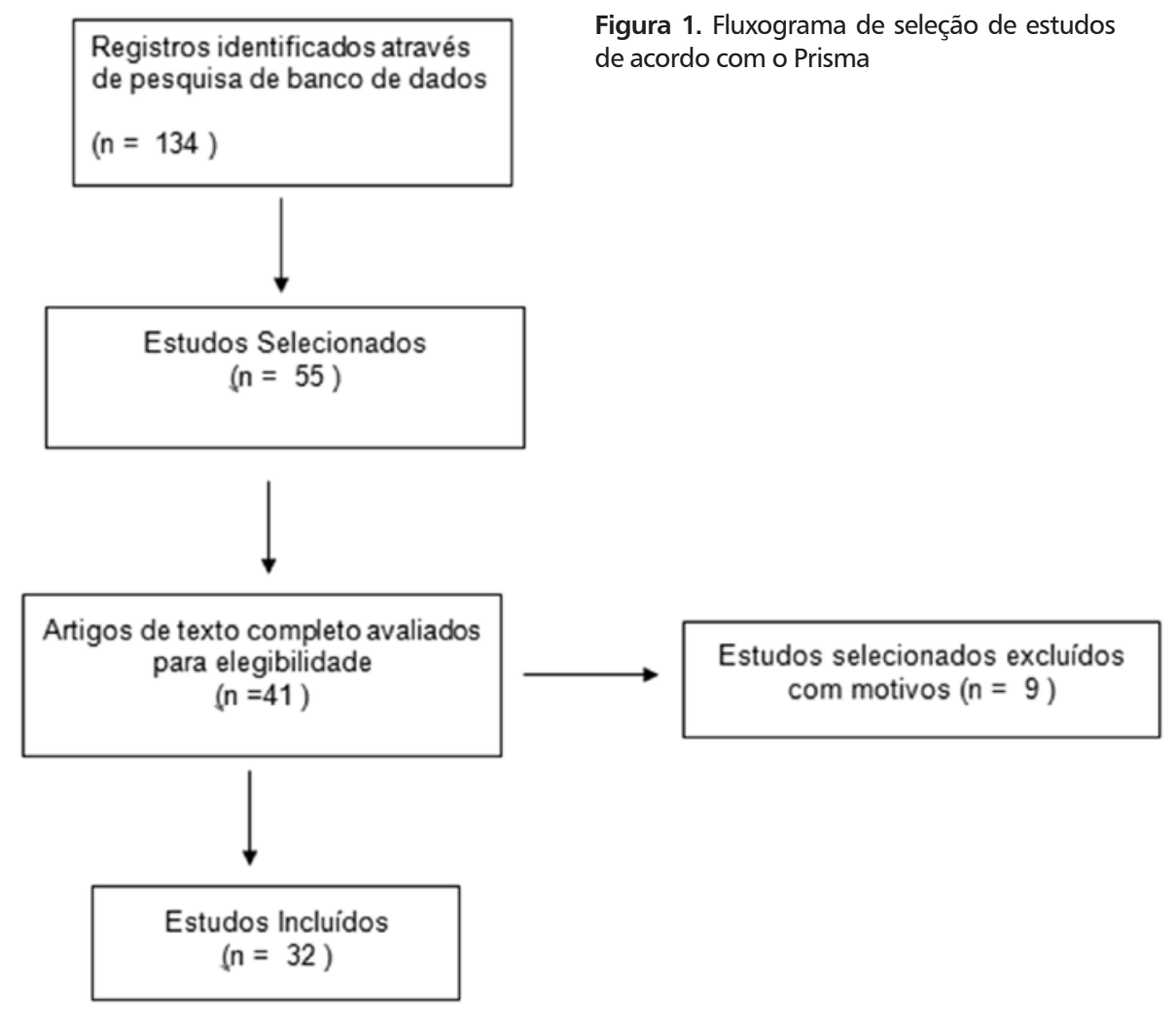

Resultados

Inicialmente foram encontradas 134 publicações utilizando as estratégias de busca anteriormente citadas, sendo 25 na Dentistry \& Oral Sciences; 70 na Pubmed e 39 na Cochrane Trials. Após a leitura dos títulos e resumos e aplicados os critérios de inclusão e exclusão, 41 publicações foram selecionadas. Nove publicações foram excluídas, após a leitura completa, por não se adequarem ao tratamento da DECHc oral, restando ao todo 32 publicações. As análises e os dados acerca de cada agente utilizado, bem como o tipo de publicação para o tratamento da doença estão descritos nas tabelas 2 e 3.

Tabela 2. Publicações elegíveis para o estudo

\begin{tabular}{|l|l|l|}
\hline Publicações excluídas & 09 & \\
\hline Publicações incluídas & 32 & 12 Revisões de literatura \\
\hline & 7 Terapias Sistêmicas \\
\hline & - Pentostatina \\
\hline - Pravastatina \\
\hline - Micofenolato Mofetila \\
\hline - Metotrexato \\
\hline - Clofazimina \\
\hline - Rituximab \\
\hline 11 Terapias Tópicas \\
\hline - Budesonida \\
\hline - Dexametasona \\
\hline - Propionato de Clobetasol \\
\hline - PUVA \\
\hline - UVB \\
\hline - Tacrolimus Monohidratado + terapia periodontal \\
\hline - Talidomida \\
\hline 02 Tratamento da Hipossalivação \\
\hline - Cevimelina \\
\hline - Eletroestimulador
\end{tabular}


Tabela 3. Terapias utilizadas para o tratamento da DECHc Oral

\begin{tabular}{|c|c|c|c|c|c|}
\hline Agente usado & $\begin{array}{c}\text { Tipo de } \\
\text { publicação }\end{array}$ & $\begin{array}{l}\text { Via de } \\
\text { utilização }\end{array}$ & $\begin{array}{l}\text { Mecanismo de } \\
\text { ação }\end{array}$ & $\begin{array}{l}\text { Resposta em } \\
\text { cavidade oral }\end{array}$ & Efeitos colaterais \\
\hline $\begin{array}{l}\text { BUDESONIDA }{ }^{23,24,25} \\
\left.\text { (BUDESONID }{ }^{\circledR}\right)\end{array}$ & Ensaios Clínicos & TÓPICA & $\begin{array}{l}\text { Anti-inflamatório } \\
\text { esteroidal de alta } \\
\text { potência }\end{array}$ & $\begin{array}{c}83-53,8-100 \% \\
(N=12-26-10)\end{array}$ & $\begin{array}{l}\text { Candidíase, ardência bucal, } \\
\text { infecções oportunistas por } \\
\text { absorção sistêmica }\end{array}$ \\
\hline $\begin{array}{l}\text { PENTOSTATINA } \\
\left.\text { (NIPENT }{ }^{\circledR}\right)\end{array}$ & Ensaio Clínico & SISTÊMICA & $\begin{array}{l}\text { Inibidor de ade- } \\
\text { nosina desami- } \\
\text { nase, utilizado na } \\
\text { DECH aguda }\end{array}$ & $\begin{array}{c}62 \%(N=39) \\
R G=55 \% \\
(N=58)\end{array}$ & $\begin{array}{c}\text { Toxicidade renal, fadi- } \\
\text { ga, náuseas e vômitos. } \\
\text { Observado piora em } \\
\text { cavidade oral em } 25 \% \text { dos } \\
\text { pacientes }\end{array}$ \\
\hline $\begin{array}{l}\text { PRAVASTATINA } \\
\text { (NOVINA } \\
\left(\text { NO }^{\circledR}\right.\end{array}$ & Ensaio Clínico & SISTÊMICA & $\begin{array}{l}\text { Supressão de } \\
\text { Interferon gama. } \\
\text { Inibidor seletivode } \\
\text { adesão molecular } \\
\text { intercelular }\end{array}$ & $\begin{array}{c}27 \%(\mathrm{~N}=18) \\
\mathrm{RG}=28 \% \\
(\mathrm{~N}=18)\end{array}$ & Risco de rabdomiosite \\
\hline $\begin{array}{l}\text { DEXAMETASONA }{ }^{27} \\
\left.\text { (ACETAZONA }^{\circledR}\right)\end{array}$ & Relato de Caso & TÓPICA & $\begin{array}{l}\text { Anti-inflamatório } \\
\text { esteroidal mais } \\
\text { utilizado para } \\
\text { tratamento tópico } \\
\text { de lesões autoi- } \\
\text { munes }\end{array}$ & $\begin{array}{l}\text { Remissão } \\
\text { completa }\end{array}$ & Não relatado \\
\hline $\begin{array}{l}\text { PROPIONATO DE CLOBETASOL }{ }^{26} \\
\text { (PROPIOSOL }^{\circledR} \text { ) }\end{array}$ & Ensaio clínico & TÓPICA & $\begin{array}{l}\text { Anti-inflamatório } \\
\text { esteroidal de alta } \\
\text { potência }\end{array}$ & $85 \%(N=13)$ & Ardência bucal \\
\hline PUVA $^{19,28}$ & $\begin{array}{l}\text { Relato de caso e } \\
\text { Ensaio clínico }\end{array}$ & TÓPICA & $\begin{array}{l}\text { Exato mecanismo } \\
\text { é desconhecido. } \\
\text { Quando fotoati- } \\
\text { vado o meto-- } \\
\text { xypsoralen que } \\
\text { é conjugado ao } \\
\text { DNA causa injúria } \\
\text { celular }\end{array}$ & $\begin{array}{l}\text { Resposta com- } \\
\text { pleta }-85 \% \\
(\mathrm{~N}=7)\end{array}$ & $\begin{array}{l}\text { Aumenta as chances de } \\
\text { câncer renal e fígado }\end{array}$ \\
\hline UVB $^{10,11}$ & $\begin{array}{l}\text { Revisão de } \\
\text { literatura e série } \\
\text { de casos }\end{array}$ & TÓPICA & $\begin{array}{l}\text { Inativação de } \\
\text { células de Lan- } \\
\text { gerhans, impedin- } \\
\text { do a liberação de } \\
\text { citocinas }\end{array}$ & $\begin{array}{c}100 \%(\mathrm{~N}=1) \\
\text { Reduziu } \\
\text { sintomatologia } \\
\text { em } 100 \% \text { de } \\
\text { pacientes com } \\
\text { escleroderma } \\
\text { perioral }(\mathrm{N}=2)\end{array}$ & Sem efeitos colaterais \\
\hline $\begin{array}{l}\text { MICOFENOLATO MOFETILA } \\
\text { (MYFORTIC } \\
\text { (M) }^{\circledR} \text { ) }\end{array}$ & Ensaio clínico & SISTÊMICA & $\begin{array}{l}\text { Inferfere na sínte- } \\
\text { se de purinas nos } \\
\text { linfócitos }\end{array}$ & $\begin{array}{c}100 \%(N=6) \\
R G=60 \% \\
(N=15)\end{array}$ & $\begin{array}{l}\text { Candidíase, diarreia, náu- } \\
\text { sea, vômito e toxicidade } \\
\text { hepática }\end{array}$ \\
\hline $\begin{array}{l}\text { METOTREXATO }{ }^{16,17} \\
\left.\text { (TEVAMETHO }^{\circledR}\right)\end{array}$ & Ensaios clínicos & SISTÊMICA & $\begin{array}{l}\text { Propriedades imu- } \\
\text { nossupressivas e } \\
\text { anti-inflamatórias }\end{array}$ & $\begin{array}{c}42-75 \% \\
(\mathrm{~N}=12-4) \\
\mathrm{RG}=83 \%- \\
76,2 \% \\
(\mathrm{~N}=86-21)\end{array}$ & $\begin{array}{l}\text { Trombocitopenia, mucosi- } \\
\text { te, leucopenia e mucosite }\end{array}$ \\
\hline $\begin{array}{l}\text { CLOFAZIMINA }^{18} \\
\text { (LAMPRENE }^{\circledast} \text { ) }\end{array}$ & Ensaio clínico & SISTÊMICA & $\begin{array}{l}\text { Imunossupressão } \\
\text { secundária da } \\
\text { função de } \\
\text { células T }\end{array}$ & $\begin{array}{c}50 \%(\mathrm{~N}=6) \\
\mathrm{RG}=55 \% \\
(\mathrm{~N}=22)\end{array}$ & $\begin{array}{l}\text { Hipopigmentação em con- } \\
\text { juntiva, náusea, constipa- } \\
\text { ção e diarreia }\end{array}$ \\
\hline $\begin{array}{l}\text { TACROLIMUS MONOHIDRATADO (PRO- } \\
\text { TROPIC }^{\circledR} \text { ) + TERAPIA PERIODONTAL }{ }^{30}\end{array}$ & Relato de Caso & TÓPICA & $\begin{array}{l}\text { Imunossupressão } \\
\text { local, permitindo } \\
\text { um controle pe- } \\
\text { riodontal efetivo }\end{array}$ & $\begin{array}{l}\text { Resposta com- } \\
\text { pleta* }\end{array}$ & Sem efeitos colaterais \\
\hline $\begin{array}{l}\text { TALIDOMIDA }^{31} \\
\text { (FUNED }^{\circledR} \text { ) }\end{array}$ & Carta ao Editor & TÓPICA & $\begin{array}{l}\text { Redução dos } \\
\text { níveis de TNF } \\
\text { alfa no local de } \\
\text { aplicação }\end{array}$ & $66 \%(N=3)$ & Sem efeitos colaterais \\
\hline
\end{tabular}

:: CONTINUAÇÃO NA PÁGINA SEGUINTE :: 


\begin{tabular}{|c|c|c|c|c|c|}
\hline Agente usado & $\begin{array}{c}\text { Tipo de } \\
\text { publicação }\end{array}$ & $\begin{array}{c}\text { Via de } \\
\text { utilização }\end{array}$ & $\begin{array}{l}\text { Mecanismo de } \\
\text { ação }\end{array}$ & $\begin{array}{l}\text { Resposta em } \\
\text { cavidade oral }\end{array}$ & Efeitos colaterais \\
\hline $\begin{array}{l}\text { RITUXIMAB }{ }^{22} \\
\left(^{(M A B T H E R A}{ }^{\circledR}\right)\end{array}$ & Ensaio clínico & SISTÊMICA & $\begin{array}{l}\text { Anticorpo mono- } \\
\text { clonal anti CD20 }\end{array}$ & $\begin{array}{c}48 \%(\mathrm{~N}=21) \\
\text { Efetividade de } \\
64 \% \text { em casos } \\
\text { de escleroder- } \\
\text { ma perioral } \\
(\mathrm{N}=11) \\
\mathrm{RG}=65 \% \\
(\mathrm{~N}=38)\end{array}$ & $\begin{array}{l}\text { Insuficiência renal, } \\
\text { tremores, sepse por in- } \\
\text { fecção bacteriana, mu- } \\
\text { cosite oral e disgeusia }\end{array}$ \\
\hline
\end{tabular}

RG=Resposta Global

\section{Discussão}

O controle da DECHc em pacientes pós-transplantados constitui uma das características principais do sucesso do transplante de células tronco Hematopoiéticas (TCTH). O primeiro relato de caso dessa doença datou de $1965,{ }^{14} \mathrm{embora}$ seja observado um intervalo temporal grande entre as publicações, principalmente entre as décadas de 60 e 90 . A partir da década de 90, observa-se que o aumento de publicações foi diretamente proporcional à quantidade de procedimentos de TCTH e de pesquisas acerca dos mecanismos de rejeição do enxerto de medula óssea em pacientes com doenças malignas.

Segundo o consenso do National Institute of Health $(\mathrm{NIH})^{3}$, os critérios para indicar o tratamento sistêmico ou tópico para lesões da $\mathrm{DECH}$ são estabelecidos de acordo com a quantidade de órgãos afetados e a gravidade. Caso a doença afete três ou mais órgãos ou gravidade maior que 02 para um órgão, indica-se o tratamento sistêmico. Entretanto, o consenso considera que lesões, afetando mucosa oral, olhos e trato genital, são aptas para a terapia local, sejam associadas ou não a terapia sistêmica, alcançando resultados satisfatórios.

No presente estudo, as terapias que envolveram fármacos sistêmicos, os pacientes possuíam a doença disseminada, logo, o tratamento usado não foi escolhido para resolução dos sintomas exclusivos da DECHc oral. Apesar disso, houve resultados significativamente positivos das queixas orais. ${ }^{15,16,17}$ Diante dos dados encontrados, a quantidade de publicações acerca de agentes terapêuticos usados por via tópica ou sistêmicos não variou significativamente. Esse fato constata que lesões orais podem ter boas respostas clínicas com ambas as abordagens. A medicação tópica pode potencializar os efeitos de fármacos sistêmicos, quando associados ou alcançar os resultados esperados, no caso de manifestações brandas da doença.

Os agentes sistêmicos usados apresentaram efeitos adversos graves, os quais limitam prescrição a pacientes debilitados, como portadores de insuficiência renal e hepática, onde muitos desses efeitos estão associados a sua metabolização. ${ }^{15-18}$ As medicações tópicas apresentaram efeitos adversos locais, como infecções por Candida e herpéticas e absorção sistêmica. ${ }^{4}$

Os agentes usados no tratamento local da DECHc oral, a maioria encontrada nesse estudo foi os corticoesteroides, corroborando com a indicação do $N I H .^{3-8}$ Essa classe de fármacos possui potente efeito anti-inflamatório, imunossupressor e boa tolerância em outras doenças clínica e histopatologicamente similares.

Os efeitos colaterais relacionados à imunossupressão causada pelos esteroides estimularam estudos para uso de agentes não farmacológicos como radiação ultravioleta (PUVA e UVB). Apesar de essas modalidades terapêuticas apresentarem efeitos colaterais, estes são brandos ou nulos quando comparados a outros tratamentos; ${ }^{10,19}$ sendo conhecido o risco do surgimento de um segundo tumor primário com esta terapia. Além disso, recomenda-se um maior cuidado com o uso desse tipo de tratamento pelo risco aumentado para o câncer oral em pacientes com DECHc. ${ }^{10}$ Outra modalidade terapêutica seria o uso do laser de dióxido de carbono, pelo qual tem obtido bons resultados no controle da dor em lesões orais. Entretanto, não há evidências sobre o uso dessa modalidade terapêutica no NIH. ${ }^{12}$

As complicações secundárias podem gerar queixas importantes aos pacientes com DECHc oral. A hipossalivação, cáries rampantes, xerostomia, trismo e esclerose de áreas periorais, constituem sinais e sintomas que os profissionais de saúde devem estar atentos e conhecer as maneiras de minimizá-los. Dentre os agentes usados para reduzir os sintomas da hipossalivação, foi encontrado um agente parassimpatico mimético agonista muscarínico, a cevimelina. ${ }^{20}$ Há poucas publicações acerca desse fármaco, sendo sua evidência para a terapia em DECHc derivado de outras doenças análogas. ${ }^{13}$ Segundo a conferência do NIH, é o sialogogo de escolha, junto com a pilocarpina. O uso de eletroestimulador ${ }^{21}$ não está descrito nas revisões de literatura, tão pouco nos consensos, apesar de uma série de casos alcançarem resultados significativos. Houve poucos efeitos colaterais, sua ocorrência se deu por meio do manuseio incorreto, pelo contato direto do aparelho com a mucosa. Sugerem-se ensaios clínicos randomizados, prospectivos para comprovar sua eficácia e sua viabilidade em uso clínico.

Não houve trabalhos que recomendem o manejo das lesões semelhantes à esclerodermia perioral. Segundo a indicação da NIH, aplicações de injeções intralesionais de corticoides associado à fisioterapia são preconizadas para melhora desses sintomas. Nesse estudo, o uso de Rituximab ${ }^{22}$ demonstrou melhora dessas lesões em região perioral, com uma resposta positiva em $64 \%(\mathrm{~N}=11)$ dos casos. Outras medicações sistêmicas relataram melhora nas lesões de esclerodermia, entretanto não relataram a localização. ${ }^{17-18} \mathrm{O}$ uso de UVB em uma série de relatos de caso relatou melhora dos sintomas. ${ }^{10}$ Sugere-se 
que ensaios clínicos relacionados ao tratamento da DECHc via sistêmica relacione a localização das lesões cutâneas e seu aspecto antes e após o tratamento.

A seguir serão discutidas, individualmente, as medicações tópicas encontradas nos testes clínicos selecionados na presente revisão sistemática.

\section{- Budesonida}

Budesonida é um esteroide de altíssima potência indicado para o tratamento da asma e problemas gastrointestinais. ${ }^{23}$ Há evidências de que pouca ou nenhuma supressão adrenal ocorra nas doses recomendadas. Essas características fazem com que ele seja bem tolerado para aplicações em cavidade oral. ${ }^{24} \mathrm{Um}$ estudo associou o uso de medicação tópica com e sem Budesonidapara o tratamento da DECHc oral e obteve resultado significativamente positivo de $83 \%$ para a associação em conjunto das duas vias, contra $36 \%$ de melhora para somente tratamento sistêmico. Foi relatado infecções por candidíase nos pacientes. ${ }^{23}$ As respostas aos tratamentos podem ser positivas em curto espaço de tempo, chegando até a 3 semanas. ${ }^{25}$ Um estudo comparou a eficácia dabudesonida com a dexametasona, o qual ambos reduziram a gravidade das lesões orais, entretanto esse dado não foi estatisticamente significativo. ${ }^{23}$ Seguindo o consenso da $N I H^{3}$ possui grau de recomendação $\mathrm{B}$ e evidência IIa.

\section{- Dexametasona}

A Dexametasona é a medicação de escolha para tratamento local das lesões de DECHc em cavidade oral, assim como a Prednisolona e a Triancinolona. ${ }^{13}$ Géis, pomadas e cremes são indicados para lesões localizadas devido suas propriedades hidrofóbicas, sendo as soluções indicadas para lesões generalizadas. É considerada o padrão ouro para tratamento de doenças autoimunes, sendo usada para avaliar parâmetro de eficácia em ensaios clínicos. ${ }^{19,24,26}$ Foi encontrado um relato de caso de tratamento tópico da DECHc oral. ${ }^{27}$ Dentre os efeitos adversos observados encontramos o desenvolvimento de infecções oportunistas, principalmente as fúngicas, sendo necessário uso profilático de Nistatina (100.000 UI por ml 4 vezes ao dia). ${ }^{24}$ Possui grau de recomendação A e evidência III segundo a NIH. ${ }^{8}$

\section{- Propionato de Clobetasol}

O Clobetasol é um anti-inflamatório esteroidal mais potente que a Dexametasona, sendo utilizado na forma de gel a $0,05 \% .{ }^{26}$ Possui recomendação A e evidência Ia. ${ }^{8}$ Foi encontrado um estudo randomizado duplo cego comparando os efeitos do Clobetasol e a Dexametasona, com excelentes resultados na amostra. ${ }^{26}$ Houve pelo menos remissão parcial em $85 \%$ $(\mathrm{N}=14)$ dos pacientes com uso de Clobetasol, comparados com o grupo Dexametasona em 33\% ( $\mathrm{N}=11)$. Os efeitos colaterais foram os mesmos qualitativamente nos dois grupos. São necessárias mais pesquisas desse tipo para que seu uso seja incluso em protocolos clínicos.

\section{- PUVA}

A radiação ultravioleta tem propriedades imunomoduladoras quando associada ao Metoxipsoralen. Esse fármaco é administrado intraoralmente a $0,3 \mathrm{mg} / \mathrm{kg}, 1$ hora antes da aplicação de radiação ultravioleta A. Seu exato mecanismo de ação não é conhecido, ocorrendo possivelmente uma indução à resposta inflamatória e melanização por proliferação da camada córnea da epiderme. ${ }^{28} \mathrm{~A}$ indicação para esse tratamento é a segunda linha de tratamento, ou seja, as lesões orais foram refratárias às terapias com corticoides. Uma publicação encontrada comparando a terapia do PUVA com outros esteroides revelou melhora significativa com a fototerapia, entretanto os estudos apresentam amostras pequenas. ${ }^{19}$

Algumas observações devem ser seguidas para esse tratamento, as quais podem minimizar potenciais complicações. Dentre elas a luz deve ser idealmente adequada ao trabalho; para um resultado efetivo do tratamento é necessário muitas sessões de terapia. Alertas importantes aos pacientes submetidos a essa terapia são mais chances de desenvolver carcinoma espinocelular e melanoma; efeitos colaterais como náusea, hepatotoxicidade, riscos oculares são comuns às reações do metoxipsoralen. ${ }^{29}$ Apesar disso, o tratamento é bem tolerado e de fácil monitoramento; entretanto, os pacientes devem ser acompanhados em intervalos curtos para observação de possíveis efeitos colaterais. O consenso de $2015,{ }^{8}$ estabelece o uso dessa terapia apenas para lesões em pele com ausência de erosões ou eritemas com recomendação C e grau de evidência científica IIb. Há expectativa para inclusão de protocolos com radiação ultravioleta para o tratamento de lesões intraorais em consensos futuros.

\section{- UVB}

A fototerapia com radiação ultravioleta B inibe a resposta proliferativa a linfócitos T e células dendríticas. Nos dois estudos, envolvendo terapia com UVB, ${ }^{10,11}$ as lesões atróficas intraorais melhoraram significativamente, bem como os sintomas subjetivos tais quais desconforto oral e a dificuldade de falar e comer. Em um ensaio clínico, ${ }^{11}$ foi observada melhora na viscoelasticidade perioral, aliviando sintomas da dermoesclerose. Assim como a terapia com PUVAé considerada segunda linha de tratamento, devendo ser avaliada em mais estudos para garantir benefícios. As vantagens sobre o PUVA são seu baixo potencial carcinogênico, além de não possuir nenhum efeito colateral sistêmico. O uso dessa terapia é indicado em casos de lesões cutâneas, não sendo relatado o uso segundo o último consenso para lesões orais. Possui recomendação $\mathrm{C} e$ evidência IIa. ${ }^{8,9}$ 


\section{- Tacrolimo Monoidratado}

O uso do tacrolimus tem sido prescrito para o tratamento da DECHc cutânea; sua boa resposta para lesões orais, permitiu que fossem estudados para lesões em mucosas. Um relato de caso ${ }^{30}$ associou o uso do tacrolimus e raspagem periodontal em pacientes com $\mathrm{DECHc}$ em gengiva e descreveram que a terapia periodontal permitiu um adequado controle de placa bacteriana reduzindo as queixas álgicas; o tacrolimus agiu como um inibidor inflamatório, favorecendo a cicatrização na região. Seu uso deve ser descontinuado assim que possível, devido ao seu efeito carcinogênico, principalmente encontrado no tratamento de lesões cutâneas. Seu uso é indicado nas formas de bochecho ou em gel, segundo o consenso do NIH, possui grau de recomendação B e evidência IIa. ${ }^{8-12}$

\section{- Talidomida}

O uso de Talidomida não é recomendado para o tratamento da DECHc oral. Seu uso é experimental, devendo ser usada apenas para ensaios clínicos ou casos individuais. ${ }^{12}$ Foi encontrado uma publicação tipo carta ao editor relatando uma melhora em dois pacientes com padrão liquenoide da DECHc oral. ${ }^{30}$ Seu mecanismo de ação por inibição de TNF alfa pode ser útil para futuras pesquisas. Não foram encontrados recomendações e evidências de uso nos consensos para terapia em cavidade oral. ${ }^{8}$

\section{Conclusão}

O tratamento da DECHc é desafiador para os profissionais de saúde. Por se tratar de uma complicação pós-TCTH, os pacientes são acompanhados por uma equipe multidisciplinar. As terapias sistêmicas e tópicas com imunossupressores são largamente difundidas na literatura com bons resultados clínicos. É importante que essa área do conhecimento seja compartilhada nas faculdades de Odontologia para que o cirurgião-dentista possa colaborar no tratamento e controle desses pacientes.

\section{Referências ::}

1.Mays JW, Fassil H, Edwards DA, Pavletic SZ, Bassim CW.Oral chronic graft versus host disease: current pathogenesis, therapy and research. Oral Dis. 2013;19(4):327-46.

2.Treisler NS, Woo SB, O’Holleran EW, Lehmann LE, Parsons SK, Guinan EC.Oral chronic graft-versus-host disease inpediatric patients after hematopoietic stem cell transplantation.Biol Blood Marrow Transplant. 2005;11(9):721-31.

3. Jagasia MH, Greinix HT, Arora M, Williams KM, Wolff D, Cowen EW, et al. National Institutes of Health Consensus Development Project on Criteria for Clinical Trials in Chronic Graft-versus-Host Disease: I. The 2014 Diagnosis and Staging Working Group report. Biol Blood Marrow Transplant. 2015;21(3):389-401.e1.

4. Meier JK, Wolff D, Pavletic S, Greinix H, Gosau M, Bertz H, et al. Oral chronic graft-versus-host disease: report from the International Consensus Conference on clinical practice in cGVHD. Clin Oral Investig. 2011;15(2):127-39.

5. Horwitz ME, Sullivan KM. Chronic graft-versus-host disease. Blood Rev. 2006;20(1):15-27.

6. Woo SB, Lee SJ, Schubert MM.Graft-vs.-host disease.CritRev Oral Biol Med. 1997;8(2):201-16.

7. Lee SJ, Flower ME. Recornizing and managing chronic graft versus host disease.Hematology Am Soc Hematol Educ Program. 2008:134-41.

8. Carpenter PA, Kitko CL, Elad S, Flowers ME, Gea-Banacloche JC, Halter JP. National Institutes of Health Consensus Development Project on Criteria for Clinical Trials in Chronic Graft-versus-Host Disease: V. The 2014 Ancillary Therapy and Supportive Care Working Group Report. Biol Blood Marrow Transplant. 2015;21(7):1167-87.

9. Imanguli MM, Alevizos I, Brown R, Pavletic SZ, Atkinson JC.Oral graft-versus-host disease.Oral Dis. 2008;14(5):396-412.

10. Elad S, Garfunkel AA, Enk CD, Galili D, Or R.Ultraviolet B irradiation: a new therapeutic concept for the management of oral manifestations of graft-versus-host disease. Oral Surg Oral Med Oral Pathol Oral RadiolEndod. 1999;88(4):444-50.

11. Enk CD, Elad S, Vexler A, Kapelushnik J, Gorodetsky R, Kirschbaum
M.Chronic graft-versus-host disease treated with UVB phototherapy. Bone Marrow Transplant. 1998;22(12):1179-83.

12. Elad S, Or R, Shapira MY, Haviv A, Galil D, Garfunkel AA, et al. CO laser in oral graft-versus-host disease: a pilot study Bone Marrow Transplantation. 2003;32:1031-4.

13. Inamoto Y, Martin PJ, Chai X, Jagasia M, Palmer J, Pidala J, et al. Clinical benefit of response in chronic graft-versus-host disease. Biol Blood Marrow Transplant. 2012;18(10):1517-24.

14. Hathaway WE, Githens JH, Blackburn WR, et al. Aplastic anemia, histiocytosis and erythrodermia in immunologically deficiente children Engi J Med. 1965;273:953-8.

15. Jacobsohn DA, Chen AR, Zahurak M, Piantadosi S, Anders V, Bolaños-Meade J, et al. Phase II study of pentostatin in patients with corticosteroid-refractory chronic graft-versus-host disease. J Clin Oncol. 2007;25(27):4255-61.

16. Huang XJ, Jiang Q, Chen H, Xu L, Liu D, Chen Y, et al. Low-dose methotrexate for the treatment of graft-versus-host disease after allogeneic hematopoietic stem cell transplantation.Bone Marrow Transplant. 2005;36(4):343-8.

17. Wang Y, Xu LP, Liu DH, Chen H, Chen YH, Han W, et al. First-line therapy for chronic graft-versus-host disease that includes low-dose methotrexate is associated with a high response rate. Biol Blood Marrow Transplant. 2009;15(4):505-11.

18. Lee SJ, Wegner SA, McGarigle CJ, Bierer BE, Antin JH. Treatment of chronic graft-versus-host disease with clofazimine. Blood. 1997;89(7):2298-302.

19. Wolff D, Anders V, Corio R, Horn T, Morison WL, Farmer E, et al. Oral PUVA and topical steroids for treatment of oral manifestations of chronic graft-vs.-host disease. Photodermatol Photoimmunol Photomed. 2004;20(4):184-90.

20. Carpenter PA, Schubert MM, Flowers ME.Cevimeline reduced mouth dryness and increased salivary flow in patients with xerostomia complicating chronic graft-versus-host disease. Biol Blood Marrow Transplant. 2006;12(7):792-4. 
21. Zadik Y, Zeevi I, Luboshitz-Shon N, Dakwar N, Wolff A, Shapira MY, et al. Safety and efficacy of an intra-oral electrostimulator for the relief of dry mouth in patients with chronic graft versus host disease: Case series. Med Oral Patol Oral Cir Bucal. 2014;19(3):e212-9.

22. Zaja F, Bacigalupo A, Patriarca F, Stanzani M, Van Lint MT, Filì C,et al. Treatment of refractory chronic GVHD with rituximab: a GITMO study. BoneMarrowTransplant. 2007;40(3):273-7.

23. Sari I, Altuntas F, Kocyigit I, Sisman Y, Eser B, Unal A, et al. The effect of budesonide mouthwash on oral chronic graft versus host disease.Am J Hematol. 2007;82(5):349-56.

24. Park AR, La HO, Cho BS, Kim SJ, Lee BK, Rhie JY, et al. Comparison of budesonide and dexamethasone for local treatment of oral chronic graft-versus-host disease. Am J Health SystPharm. 2013;70(16):1383-91.

25. Elad S, Or R, Garfunkel AA, Shapira MY. Budesonide: a novel treatment for oral chronic graft versus host disease. Oral Surg Oral Med Oral Pathol Oral RadiolEndod. 2003;95(3):308-11.

26. Noce CW, Gomes A, Shcaira V, Corrêa ME, Moreira MC, Silva Júnior A, et al. Randomized double-blind clinical trial comparing clobetasol and dexamethasone for the topical treatment of symptomatic oral chronic graft-versus-host disease. BiolBloodMarrowTransplant. 2014;20(8):1163-8.

27. Stoopler ET. Management of oral GVHD. J Can Dent Assoc. 2013;79:d37.

28. Redding SW, Callander NS, Haveman CW, Leonard DL.Treatment of oral chronic graft-versus-host disease with PUVA therapy: case report and literature review.Oral Surg Oral Med Oral Pathol Oral RadiolEndod. 1998;86(2):183-7.

29. Mitchell SA, Jacobsohn D, Thormann Powers KE, Carpenter PA, Flowers ME, Cowen EW, et al. A multicenter pilot evaluation of the National
Institutes of Health chronic graft-versus-host disease (cGVHD) therapeutic response measures: feasibility, reliability, interrater and minimum detectable change. BiolBloodMarrowTransplant. 2011;17(11):1619-29.

30. Conrotto D, Broccoletti R, Carcieri P, Giaccone L, Arduino PG. Topical tacrolimus and periodontal therapy in the management of case of oral chronic GVHD characterized by specific gingival localization. Case Rep Dent. 2014;2014:127219.

31. St John L, Gordon SM, Childs R, Marquesen M, Pavletic SZ, Wu TX et al.Topical thalidomide gel in oral chronic graft-versus-host disease and role of in situ cytokine expression in monitoring biological activity.BoneMarrowTransplant. 2013;48(4):610-11.

32. Busca A, Saroglia EM, Lanino E, Manfredini L, Uderzo C, Nicolini $\mathrm{B}$, et al. Mycophenolate mofetil (MMF) as therapy for refractory chronic GVHD (cGVHD) in children receiving bone marrow transplantation. BoneMarrowTransplant. 2000;25(10):1067-71.

33. Couriel D, Carpenter PA, Cutler C, Bolaños-Meade J, Treister NS, Gea-Banacloche J,et al.Anciilary therapy and supportive care of chronic graft versus host disease: NIH consensus developed project on criteria for clinical trials in chronic graft versus host disease.BiolBloodMarrowTransplant. 2006;12(4):375-96.

34. Busca A, Locatelli F, Vai S, Dall'Omo AM, Gar-giulo A, Falda M. Clinical grading of oral chronic graft-versus-host disease in 104 consecutive adult patients. Haematologica. 2005;90(4):567-9.

35. da Fonseca MA, Hong C. A overview of chronic oral graft host disease following pediatric hematopoietic stem cell transplantation. PediatrDent. 2008 Mar;30(2):98-104

36. Hori A, Kanda Y, Goyama S, Onishi Y, Komeno Y. A prospective trial to evaluate the safety and efficacy of pravastatin for the treatment of refractory chronic graft-versus-host disease.Transplantation. 2005;79(3):372-4.

Recebido em: 26/04/2016 / Aprovado em: 12/06/2016

Felipe Souza Lima Alencar

Rua do Matoso, 42/401, Tijuca

Rio de Janeiro/RJ, Brasil - CEP: 20270-130

E-mail: felipe.alencar503@gmail.com 